\title{
The Choice of AC Electricity Bridge Adjustment Parameters
}

\author{
Zaiqi Fu, Changji Shan, Yuhui Luo \& Xungang Yang \\ Department of Physics \\ Zhaotong Teacher's College \\ Zhaotong 657000, China
}

Tel: 86-870-215-3826 E-mail:zhxfzq@126.com

\begin{abstract}
It is set forth that how to choose adjustment parameters in the plural plane coordinate figure by the relation of Electricity Bridge's balance condition and adjustment parameters.

Keywords: Adjustment parameters, Choice, Balance

On the discussion of $\mathrm{AC}$ electricity bridge in some schoolbooks and correlated datum, it has been put forward that how to choose some quantities as adjustment parameters or the selected adjustment parameters from the observed quantities which have been discriminated reading. The new viewpoint is brought forward that how to choose the adjustment parameters by the point of view of adjustment velocity.
\end{abstract}

\section{The Balance Condition of AC Electricity Bridge}

The balance process of $A C$ electricity bridge is decided by the change of eqn(1).Because $Z_{1}, Z_{2}, Z_{3}, Z_{4}$ are composed of resistance,apacitance and inductance which are parallel connection or in series,xcept the observed parameters, the others quantities can be regarded as adjustment parameters.

$\mathrm{P}=\mathrm{Z}_{2} \mathrm{Z}_{3}-\mathrm{Z}_{1} \mathrm{Z}_{4}=\mathrm{a}+\mathrm{jb}$

It is shown by eqn(1) that the two quantities at least are chosen as adjustment parameters for a and $b=0$ together.If three or the more quantities are chosen, hen adjustment times is nimiety,he balance process can be very much trouble.In the most things, two quantities are chosen as adjustment parameters.

The selections have more groups.Some groups can not attain the balance of electricity bridge;some groups may attain the aim,but nimiety.It is the best choice that not only some groups may attain the balance but also the adjustment times are few.The article will set forth how to choose correct adjustment parameters quickly through the frequent used AC electricity bridge.

\section{The Quickly Chosen of AC Electricity Bridge Adjustment Parameters}

At the balance point of electricity bridge,the relation of $a, b$ and adjustment parameters may be expressed by parallel beeline family ${ }^{[3]}$. The more nearly $90^{\circ}$ of two parameter beeline angle, the more quickly of balance speed. It can be shown that which parameter beeline are perpendicularity or parallelism one another. The best choice is two parameters of perpendicularity, not parallelism. Without parameters of perpendicularity, the best seemliness choice is two parameters which angle is rather big.

\subsection{Capacitance Electricity Bridge}

According to Fig.2,it is shown the expression formula of $a$ and $b$ are eqn(2) and eqn(3) separated by the eqn(1).The beelines of each parameters are shown in Fig.3.It is the best choice that $R_{2}$ and $C_{2}$ are adjustment parameters ,as shown in Fig.3.

$$
\mathrm{a}=\mathrm{R}_{\mathrm{x}} \mathrm{R}_{3}-\mathrm{R}_{4} \mathrm{R}_{2} \quad \text { (2) } \quad \mathrm{b}=\frac{R_{4}}{C_{2} \omega}-\frac{R_{3}}{C_{x} \omega}
$$

\subsection{Inductance Compare Electricity Bridge}

According to Fig.4,it is shown the expression formula of $a$ and $b$ are eqn(4) and eqn(5) separated by the eqn(1).The beelines of each parameters are shown in Fig.5.It is the best choice that $\mathrm{R}_{2}$ and $\mathrm{L}_{2}$ are adjustment parameters ,as shown in Fig.5.

$$
\mathrm{a}=\mathrm{R}_{\mathrm{x}} \mathrm{R}_{3}-\mathrm{R}_{2} \mathrm{R}_{4}
$$

$$
\mathrm{b}=\mathrm{L}_{\mathrm{x}} \mathrm{R}_{3} \omega-\mathrm{L}_{2} \mathrm{R}_{3} \omega
$$

\subsection{Schering Electricity Bridge}

According to Fig.6,it is shown the expression formula of $a$ and $b$ are eqn(6) and eqn(7) separated by the eqn(1).The beelines of each parameters are shown in Fig.7. Because the angle of $R_{3}$ and $C_{3}$ is rather big,it is the best choice that $R_{3}$ and $C_{3}$ are 
adjustment parameters ,as shown in Fig.7.

$$
\begin{aligned}
& \mathrm{a}=\frac{R_{x} R_{3}}{1+\left(R_{3} C_{3} \omega\right)^{2}}-\frac{R_{3}^{2} C_{3} \omega}{C_{x} \omega\left[1+\left(R_{3} C_{3} \omega\right)^{2}\right]} \\
& \mathrm{b}=\frac{R_{2}}{C_{4} \omega}-\frac{R_{x} R_{3}^{2} C_{3} \omega}{1+\left(R_{3} C_{3} \omega\right)^{2}}-\frac{R_{3}}{C_{x} \omega\left[1+\left(R_{3} C_{3} \omega\right)^{2}\right]}
\end{aligned}
$$

\subsection{Maxwell Electricity Bridge}

According to Fig.8, it is shown the expression formula of $a$ and $b$ are eqn(8) and eqn(9) separated by the eqn(1). The beelines of each parameters are shown in Fig.9.Because the angle of $\mathrm{R}_{3}$ and $\mathrm{C}_{3}$ is rather big,it is the best choice that $\mathrm{R}_{3}$ and $\mathrm{C}_{3}$ are adjustment parameters , as shown in Fig.9.

$$
\mathrm{a}=\frac{R_{3}\left(R_{x}+R_{3} C_{3} \omega^{2} L_{x}\right)}{1+\left(R_{3} C_{3} \omega\right)^{2}}-R_{2} R_{4} \quad \text { (8) } \quad \mathrm{b}=\frac{R_{3}\left(L_{x} \omega-R_{x} R_{3} C_{3} \omega\right)}{1+\left(R_{3} C_{3} \omega\right)^{2}}(9)
$$

For the complex AC Electricity Bridge, it is difficult to ascertain the relation which a and $\mathrm{b}$ change with some parameter varying. At the time, it may be judged by the software of origin.

\section{Conclusion}

First, the each parameters are done in the coordinate chart of a and $\mathrm{jb}$, then according to the angle between the beelines, it is a convenience method to choose perpendicularity or bigger angle as the adjustment parameter.

\section{References}

Chen, Tianxiang. (2005).High Precision tan $\delta$ Online Measure on Electricity Bridge's Balance Principle. High Voltage Technique.vol.31(1).pp.42-44

Chen, Xin.(2004).The Improvement of AC Electricity Bridge Experiment.College Physics Experiment. vol.17(4).pp.9 -11 .

Li, Yao.( 2002).The balance of Adjustment AC Electricity Bridge Quickly. College Physics Experiment. vol.15(3).pp.38-39

Liang, Bo. (2006).The Neglect Question In the Schering Electricity Bridge's Testing.The Chinese Testing technique.vol.3 (22).pp.90-92

Lin, Lijuan.(2006).Making Use of Ray Separate Magnifing signal To Design the AC Electricity Bridge. College Physics.vol.25 (2).pp.43-45

Liu, Yun.(2003).The Analysis and Application In the Electricity Bridge Sensitivity and Bridge Circuitry. Journal of Shan Xi Science College.vol.21(5).pp.56-58

Ou, Xinda.(2004).The relation of Schering Electricity Bridge's Testing Range and Location Selection. Northwest Electric Power Technique.vol.3.pp.95-96

Qin,Zhenhuang. (2003).Electrotechnician,Beijing: China Higher Education Press.

Yang,Shu-wu. (2000).Common Physics Experiment,Beijing: China Higher Education Press.

Zhang, Gongming.(2008).The discussion of Electricity Bridge Characteristic and Definition.Chinese Measurement.vol. 2.pp.69-71

Zhang, Jing.(2005).The parameter measure and Electricity Bridge meter on L-C-R. Electron Quality. Testing Technique .vol.7.pp.15-16

Zheng, Jiaxiang \&Lu, Yuxin. (1982).Electron Measure Principle.Beijing: National Defence Industry Press. 


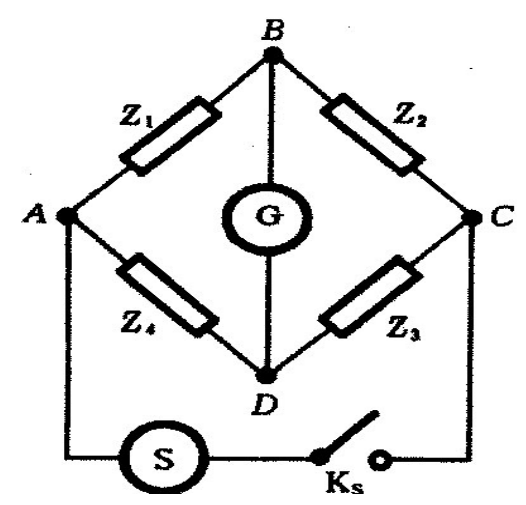

Figure 1. Electricit Bridge Circuitry

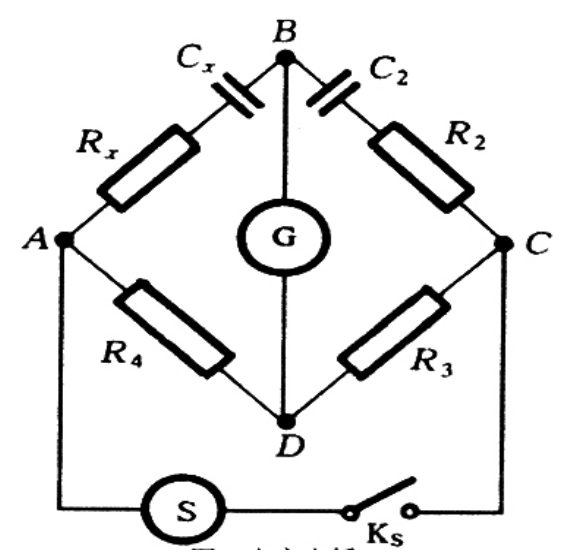

Figure 2. Capacitance electricity bridge

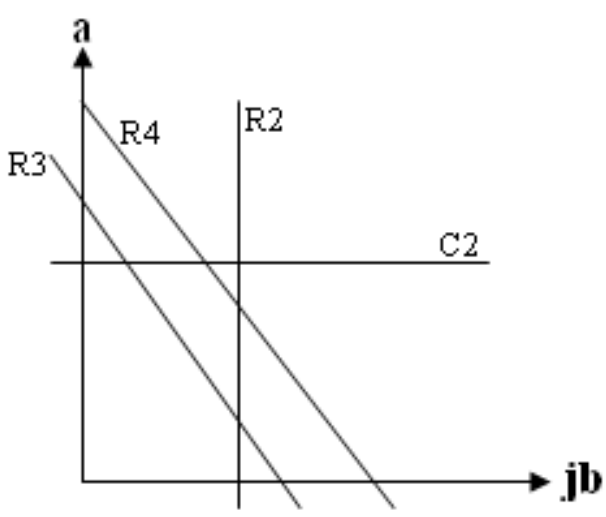

Figure 3. The beeline chart of Capacitance electricity bridge parameter 


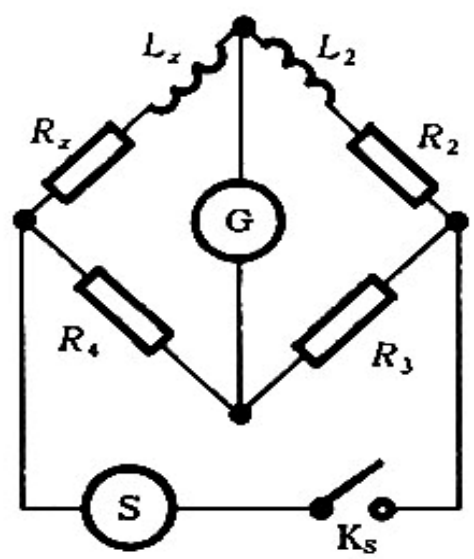

Figure 4. Inductance Compare Electricity Bridge

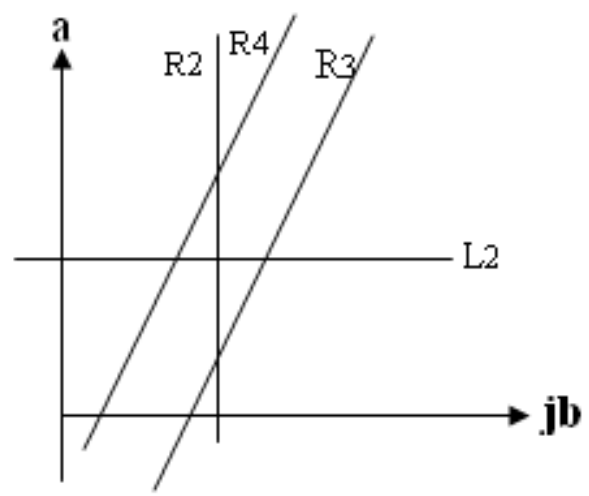

Figure 5. The beeline chart of Inductance Compare bridge parameter

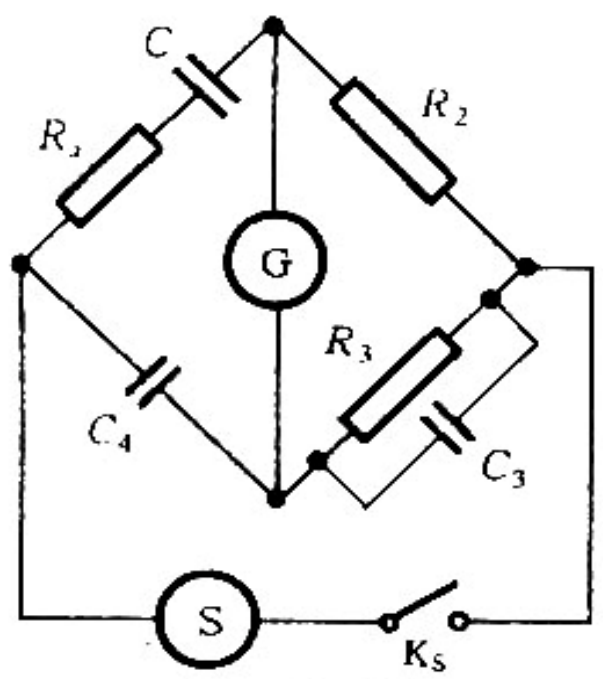

Figure 6. Schering Electricity Bridge 


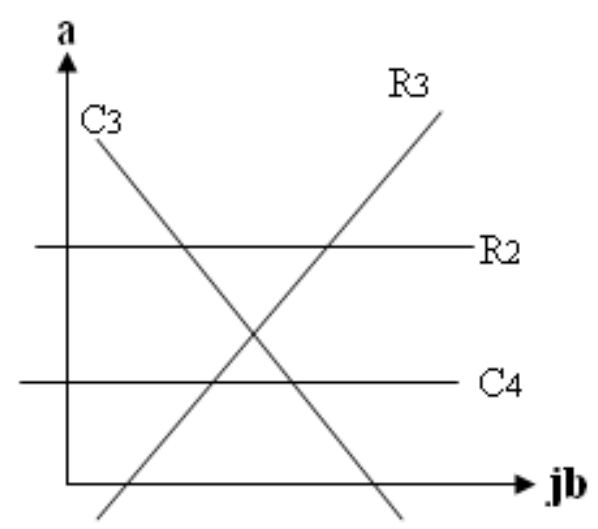

Figure 7. The beeline chart of Schering Electricity Bridge Parameter

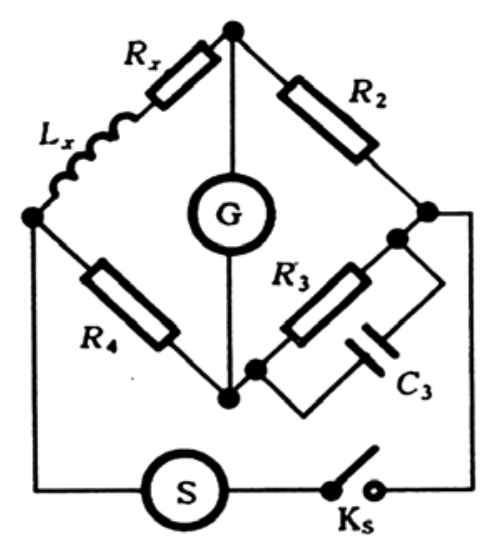

Figure 8. Maxwell Electricity Bridge

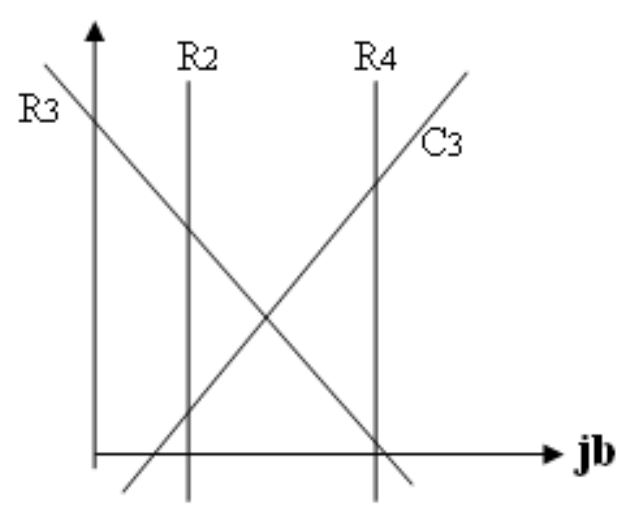

Figure 9. The beeline chart of Maxwell Electricity Bridge Parameter 